\title{
Decoupling Salt- and Polymer-Dependent Dynamics in Polyelectrolyte Complex Coacervates via Salt Addition
}

\author{
Frances J. Morin ${ }^{1}$, Marissa L. Puppo ${ }^{1}$, and Jennifer E. Laaser ${ }^{1,2}$
}

\begin{abstract}
In polyelectrolyte complex coacervates, changes in salt concentration and changes in polymer concentration are typically strongly coupled, complicating interpretation of the salt- and polymerconcentration-dependent dynamics of these materials. To address this problem, we developed a "salt addition" method for preparation of complex coacervates that allows the salt concentration of a coacervate sample to be varied without changing its polymer concentration. This method was used to prepare coacervates of poly(styrene sulfonate) (PSS) with poly(diallyldimethylammonium chloride) (PDADMAC) with salt concentrations between 1.2 and $2 \mathrm{M}$ and volume fractions of polymer between 0.1 and 0.25 . Characterization of these samples by small-amplitude oscillatory shear rheology revealed that the relaxation times scale significantly more strongly with polymer volume fraction than has been previously assumed, highlighting the need to account for both salt and polymer-dependent contributions to the dynamics of these complex materials.
\end{abstract}

\footnotetext{
${ }^{1}$ Department of Chemistry, University of Pittsburgh

${ }^{2}$ To whom correspondence should be addressed: j.laaser@pitt.edu
} 


\section{Introduction}

Polyelectrolyte complex coacervates are a versatile class of polymer-rich liquids formed by associative phase separation of oppositely-charged polymers in aqueous solution. ${ }^{1,2}$ These materials are typically viscoelastic, and can range from gel-like elastic solids to viscous liquids depending on the polymer chemistry and solution conditions. ${ }^{2-5}$ Controlling these materials properties is critical for a wide range of applications, ranging from drug and protein delivery to self-healing materials. ${ }^{6-10}$ Developing a detailed fundamental understanding of the physical origins of viscoelasticity in these materials presents an important challenge to inform rational design of functional coacervate materials.

To date, the "sticky Rouse" model has emerged as the most viable theoretical framework describing relaxation processes in complex coacervates. ${ }^{4,11}$ In the sticky Rouse model, the polymer chains are assumed to interact via "sticky" interactions between specific sites on the polymer chains. These stickers can, on some characteristic timescale, separate and find new partners, allowing the material to relax. ${ }^{12}$ In its most general form, this model predicts that the longest relaxation time, $\tau_{\max }$, of the material goes as

$$
\tau_{\text {max }} \sim N^{2} e^{E_{a} / k T} \phi_{\text {pol }}^{\beta}
$$

where $N$ is the degree of polymerization of the polymer chains, $E_{a}$ is the activation energy for rearrangement of each sticker pair, $\phi_{p o l}$ is the volume fraction of polymer, and $\beta$ is an exponent describing the concentration dependence of the dynamics. ${ }^{12,13}$ When applied to complex coacervates, the sticky interactions are assumed to arise from interactions of oppositely-charged ions on different polymer chains. ${ }^{4,11}$ Because the activation energy for rearrangement, and thus the lifetime of these ion pairs can depend on the presence of other ions in solution (which may either screen the coulombic interactions between the charged sites on the polymer chains, ${ }^{11}$ or may serve

as extrinsic partners for ion exchange ${ }^{14}$ ), the sticky Rouse treatment predicts that increasing the 
salt concentration should speed relaxation of complex coacervates. ${ }^{11,15}$

The first successful quantitative application of this model to complex coacervates was published in 2010 by Spruijt et al., who demonstrated that coacervates of poly(dimethylaminoethyl methacrylate) (DMAEMA) and poly(acrylic acid) (PAA) appeared to exhibit time-salt superposition, indicating that all of the important relaxation processes in the coacervates had the same salt dependence. ${ }^{11,15}$ Analyzing the resulting shift factors suggested that the relaxation times were well-described by the sticky Rouse model, with the "sticky" interactions arising from electrostatic interactions between oppositely-charged sites on the PDAMEMA and PAA chains. ${ }^{11}$ In this analysis, the activation energy for ion pair rearrangement was assumed to follow

$$
\frac{E_{a}}{k T}=-A \sqrt{c_{\text {salt }}}+B
$$

where $c_{\text {salt }}$ is the salt concentration in the sample and $A$ and $B$ are constants related to the Bjerrum length and ionic bond distance. This functional form for the activation energy was obtained using the Debye-Huckel theory to describe the salt dependence of the electrostatic interactions within a given ion pair. ${ }^{11}$ Other coacervates have since been shown to exhibit similar time-salt superposition behavior, ${ }^{14,16-19}$ although in some systems, the salt dependence of the relaxation time appears to be better described as

$$
\frac{E_{a}}{k T} \sim c_{\text {salt }}^{\alpha}
$$

with an exponent $\alpha$ closer to 1 rather than $0.5 .^{14,16,18}$

Interestingly, Spruijt et al.'s analysis largely ignored the effect of changes in polymer concentration on the measured dynamics of the complex coacervates. ${ }^{11,15}$ However, as the salt concentration at which the sample is prepared increases, the polymer concentration in the coacervate phase typically decreases, ${ }^{20,21}$ which may significantly affect the dynamics in these systems. Recently, a number of authors have attempted to account for this effect in their analysis of salt-dependent coacervate rheology. Marciel et al. found that in coacervates of poly(lysine) and poly(glutamic acid), a factor of $\phi_{p o l}$ had to be included in the relaxation times to adequately fit the data. ${ }^{18}$ Similarly, 
Yang et al. assumed that $\tau_{\text {max }} \sim \phi_{\text {pol }},{ }^{14}$ while Ali et al. assumed that the relaxation times scaled as $\phi_{p o l}^{7 / 3}$ to account for the polymers being in the semidilute entangled regime. ${ }^{17}$ While the assumed scaling exponents in these works were all reasonable and consistent with the scaling expected for semidilute and/or entangled polymer solutions, they cover only a small range of the scaling exponents that arise in a full treatment of the sticky Rouse model, ${ }^{12,13}$ and understanding the polymer concentration dependence is a key prerequisite for quantitative analysis of the salt-dependent relaxation mechanisms in these materials. Recent molecular dynamics simulations have even suggested that in the limit in which specific ion hydration is not taken into account, salt-mediated changes in ion pair relaxation rates may not matter at all, and that changes in the relaxation time of complex coacervates prepared at different salt concentrations may arise exclusively from changes in the polymer concentration. ${ }^{22}$ Experiments capable of independently measuring the dependence of the relaxation times on the volume fractions of polymer and salt are thus critical for understanding of the relaxation mechanisms of these materials.

Here, we address this problem using a "salt-addition" method to prepare coacervates with varying salt concentrations but similar polymer content. In this method, large coacervate samples are prepared and allowed to equilibrate. The supernatant is then decanted, and varying amounts of salt are added directly to the polymer-rich coacervate phase. This method enables preparation of coacervates with nearly identical polymer concentrations but widely varying salt concentrations, which allows the polymer- and salt-dependent components of the relaxation times to be extracted without any a priori assumptions about the scaling of the relaxation times with each of these variables. We apply this method to coacervates of poly(styrene sulfonate) (PSS) with poly(diallyldimethylammonium chloride) (PDADMAC). We find that the dependence of the relaxation time on polymer volume fraction is significantly stronger than assumed in most work to date. This work reveals the key role of polymer concentration in determining relaxation timescales of complex coacervates, and suggests that polymer concentration dependence can and should be rigorously included in analyses of these systems. 


\section{Materials and Methods}

\subsection{Materials}

Poly(sodium 4-styrenesulfonate) (PSSNa, $\mathrm{M}_{\mathrm{w}}=200,000 \mathrm{~g} / \mathrm{mol}$ ) and poly(diallyldimethyl ammonium chloride) (PDADMAC, $\left.\mathrm{M}_{\mathrm{w}}=200,000-350,000\right)$ were purchased from Sigma Aldrich. Both polymers were dialyzed against MilliQ water to remove small-molecule impurities and lyophilized before use. Potassium bromide $(\mathrm{KBr})$ was purchased from Sigma-Aldrich and used as received. All samples and stock solutions were prepared using deionized water $(18.2 \mathrm{M} \Omega \cdot \mathrm{cm})$ obtained from a Synergy water purification system (Millipore Sigma).

\subsection{Sample Preparation}

Bulk coacervates were formed from mixtures of polyelectrolyte and salt stock solutions, as has been described previously. ${ }^{23}$ Briefly, stock solutions of PSSNa and PDADMAC were prepared at charged monomer concentrations of $0.5 \mathrm{M}$, and a $\mathrm{KBr}$ stock solution was prepared at a concentration of 4 M. Samples were then prepared at each starting salt concentration with a total concentration of $0.125 \mathrm{M}$ of each charged monomer and a total volume of $50 \mathrm{~mL}$ by sequential addition of PSSNa stock solution, deionized water, $\mathrm{KBr}$ stock solution, and PDADMAC stock solution. To ensure complete mixing, the samples were vortexed for 1 minute at $2000 \mathrm{rpm}$ after each addition. The samples were then centrifuged for 5 minutes at $4000 \mathrm{rpm}$, left to equilibrate for 24 hours, centrifuged again for 20 minutes, and allowed to equilibrate for a further 5 days.

To prepare samples with added salt, the supernatant was first decanted from each equilibrated coacervate sample. The coacervate phase was then divided up into 5 or 6 individual vials, each containing approximately $0.5 \mathrm{~g}$ of coacervate. Each sample was weighed, and the amount of solid $\mathrm{KBr}$ needed to reach each target salt concentration was added to the sample. The coacervate/salt mixtures were then stirred for 1 minute and allowed to equilibrate for a minimum of one week before measurement. 


\subsection{Thermogravimetric Analysis}

Thermogravimetric analysis (TGA) measurements were carried out on a Q5000 IR Thermogravimetric Analyzer (TA Instruments) using a measurement protocol adapted from Li et al. ${ }^{21}$ For each measurement, approximately $10 \mathrm{mg}$ of sample was loaded onto a platinum pan and heated to $100{ }^{\circ} \mathrm{C}$ at a rate of $20{ }^{\circ} \mathrm{C} / \mathrm{min}$. The sample was then held at $110{ }^{\circ} \mathrm{C}$ for 60 minutes to remove all excess water in the sample. The temperature was then ramped to $610{ }^{\circ} \mathrm{C}$ at a rate of $10{ }^{\circ} \mathrm{C} / \mathrm{min}$. At $610{ }^{\circ} \mathrm{C}$, the temperature was held constant for 90 minutes to remove as much residual organic material as possible. Finally, the temperature was ramped up to $680{ }^{\circ} \mathrm{C}$ at $10{ }^{\circ} \mathrm{C} / \mathrm{min}$ to complete the measurement.

\subsection{Small-Amplitude Oscillatory Shear Rheology}

Small-amplitude oscillatory shear measurements were performed on an Anton Paar MCR-301 stress-controlled rheometer using a $25 \mathrm{~mm}$ sand-blasted parallel plate geometry. In each measurement, the frequency was swept from $600 \mathrm{rad} / \mathrm{s}$ to $0.1 \mathrm{rad} / \mathrm{s}$. The strain was simultaneously increased from $1 \%$ to $100 \%$ to improve sensitivity at low frequencies. ${ }^{24}$ Amplitude sweeps from approximately $0.1 \%$ to $100 \%$ strain at $10 \mathrm{rad} / \mathrm{s}$ were used to confirm that the entire frequency sweep was in the linear viscoelastic regime (see Supporting Information). All measurements were performed at $20{ }^{\circ} \mathrm{C}$ and a gap height of $250 \mu \mathrm{m}$. To minimize evaporation, an evaporation blocker was installed on the instrument, and the measurement protocol (including equilibration, frequency sweep, and amplitude sweep) was further designed to complete in under three hours, as has been reported previously. ${ }^{11}$ 


\section{Results}

\subsection{Preparation of Coacervates via Salt Addition}

The composition of coacervates prepared via salt addition was characterized using thermogravimetric analysis (TGA). Representative TGA data for a series of coacervates prepared at an initial salt concentration of $1.2 \mathrm{M}$ are shown in Figure 1. TGA traces for the as-formed coacervate and supernatant are shown in Figure 1(a). The TGA traces for both the coacervate and the supernatant exhibit a sharp mass loss during the isotherm at $110{ }^{\circ} \mathrm{C}$ as water evaporates from the sample. The supernatant phase loses a larger fraction of its mass at this temperature than does the coacervate, reflecting the higher water content in the supernatant. Between 110 and $610{ }^{\circ} \mathrm{C}$, both samples undergo further mass loss as the organic polymer component is burned off. Here, the coacervate phase loses more mass than the supernatant, reflecting the higher polymer concentration in the coacervate. Finally, the remaining mass after the isotherm at $610{ }^{\circ} \mathrm{C}$ is attributed to the inorganic salt $(\mathrm{KBr})$.
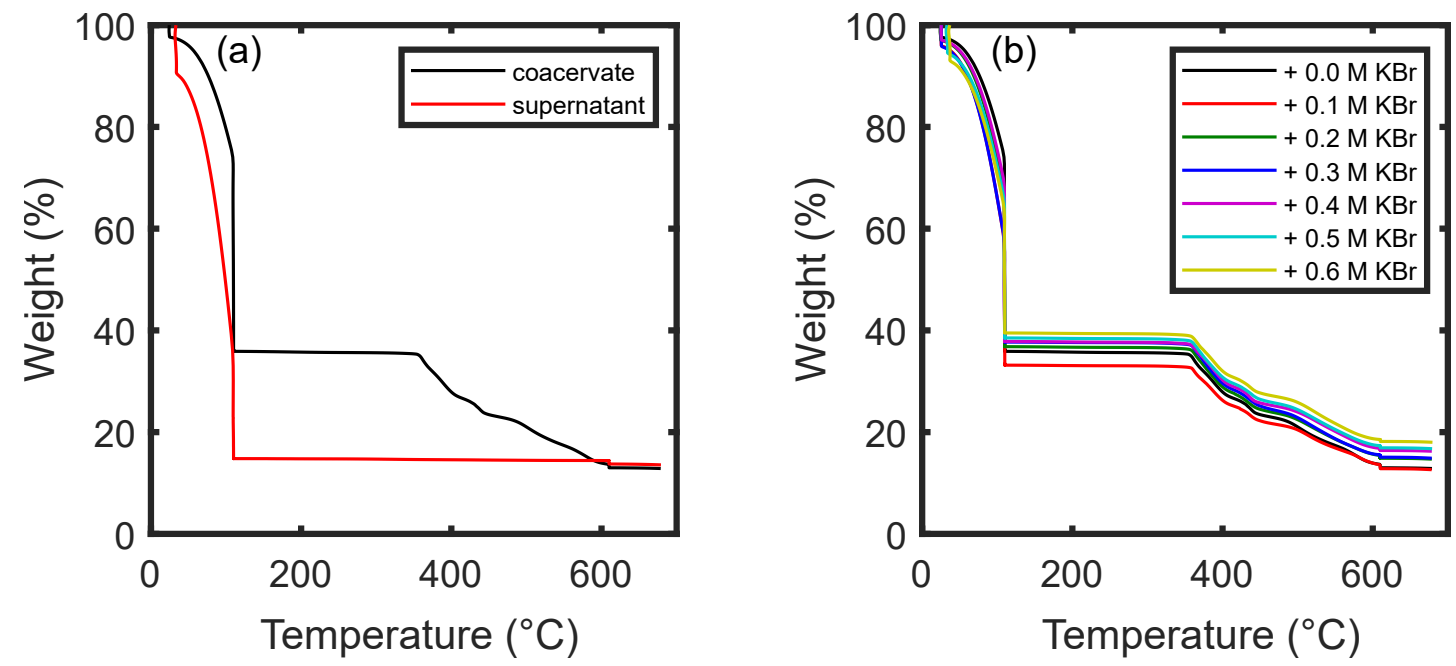

Figure 1. TGA traces for (a) the coacervate and supernatant phases of a sample prepared at $1.2 \mathrm{M}$, and (b) samples prepared by addition of solid $\mathrm{KBr}$ to the coacervate phase.

TGA traces for the coacervate samples with added salt are shown in Figure 1(b). Nominally, the mass of salt added to each sample was chosen to increase the salt concentration in increments 


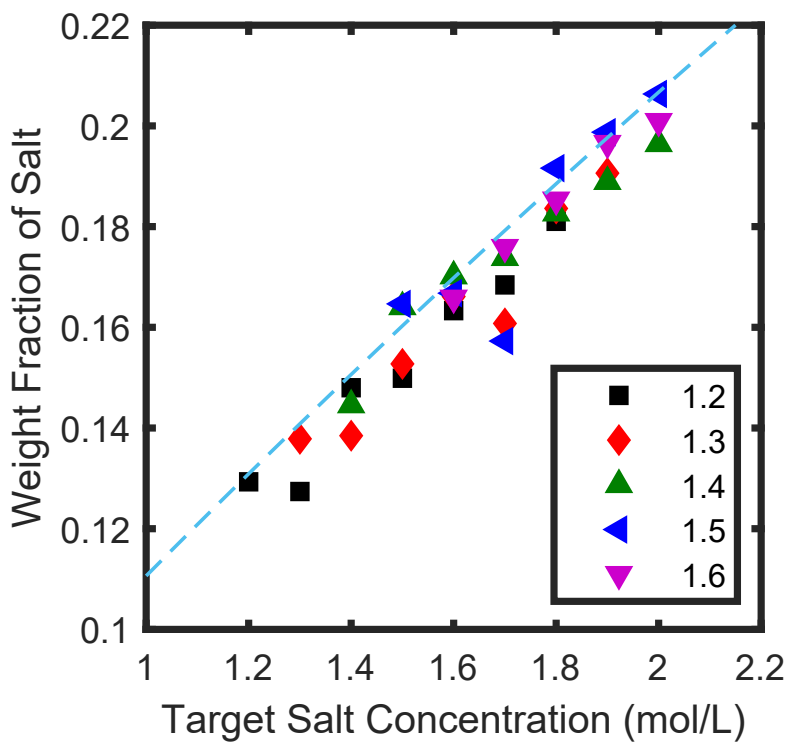

Figure 2. Comparison of the measured mass fraction of salt with the mass fraction expected for a $\mathrm{KBr}$ solution at each target salt concentration indicates that salt addition successfully reached the target salt concentrations to within $10 \%$.

of approximately $0.1 \mathrm{M}$, targeting final salt concentrations of $1.3 \mathrm{M}(1.2+0.1 \mathrm{M}), 1.4 \mathrm{M}(1.2+$ $0.2 \mathrm{M}$ ), etc., up to a maximum of $2.0 \mathrm{M}$, above which some samples became inhomogeneous. As shown in Figure 1(b), the mass remaining at the end of each TGA run did indeed increase, reflecting the increasing salt concentrations of the samples, while the mass loss in the polymer region was relatively constant. Comparison of the measured mass fraction of salt with that expected for a $\mathrm{KBr}$ solution at each target salt concentration, as shown in Figure 2, reveals that the salt addition approach was generally successful in achieving the targeted salt concentrations, with the agreement between the targeted and measured salt fractions generally within $10 \%$.

The mass fractions of polymer, water, and salt were converted to volume fractions using the bulk densities of the components $\left(1 \mathrm{~g} / \mathrm{cm}^{3}\right.$ for water, $2.75 \mathrm{~g} / \mathrm{cm}^{3}$ for $\mathrm{KBr}$, and $1.27 \mathrm{~g} / \mathrm{cm}^{3}$ for the PSS/PDADMA ion paired polymers ${ }^{25,26}$ ), as has been described previously. ${ }^{21}$ The resulting compositions are shown in Figure 3. As seen in this figure, the compositions of the as-prepared coacervate (filled circles) and supernatant (open circles) form the characteristic concave down curve typical of phase boundaries of complex coacervates..$^{20,21}$ The tie lines connecting the composition of each coacervate/supernatant pair are relatively flat, consistent with the athermal mixing 


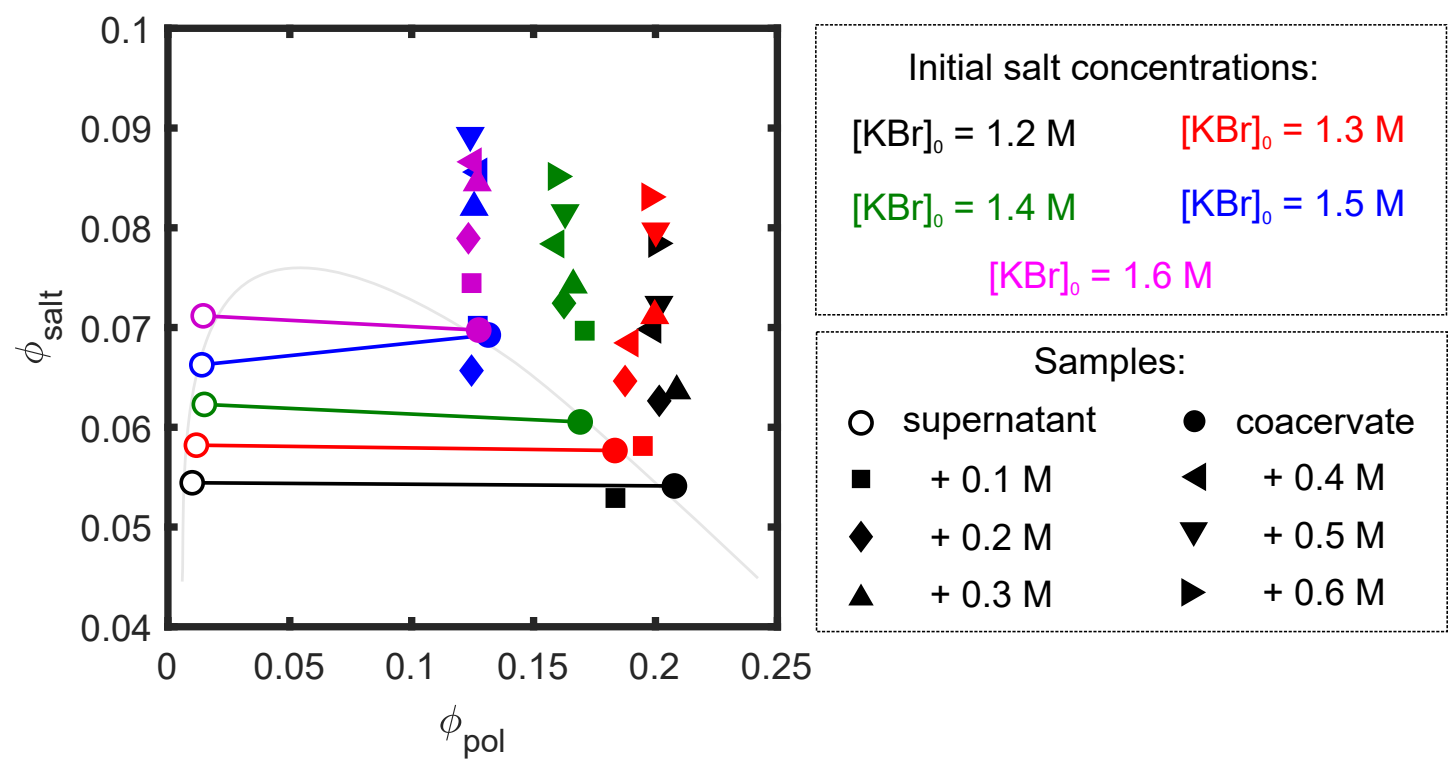

Figure 3. Compositions of as-prepared coacervates (filled circles) and supernatants (open circles), and coacervates with added salt (filled symbols). The concave-down curve connecting the asprepared coacervates is a guide to the eye indicating the shape of the phase boundary in this system.

observed in other work on PSS/PDADMAC coacervates in $\mathrm{KBr}^{26}$ The compositions of the coacervate samples with added salt generally fall on a line extending vertically above the composition of the coacervate from which they were formed, indicating that the salt addition method does indeed allow preparation of samples with an effectively fixed polymer concentration but varied salt concentrations.

\subsection{Viscoelasticity of Coacervates with Added Salt}

Frequency sweeps on the coacervate samples with and without added salt are shown in Figure 4. As seen in this figure, the coacervates formed at the lowest initial salt concentrations (1.3 and 1.4 M) exhibited a gel-like plateau, while those formed at higher salt concentrations (1.5 and $1.6 \mathrm{M})$ exhibited the characteristic $G^{\prime} \sim \omega, G^{\prime \prime} \sim \omega^{2}$ scaling expected for viscoelastic liquids in the terminal regime. The modulus of the coacervates decreased with increasing initial salt concentration, and the crossover point also shifted toward higher frequencies, as is typical for coacervate systems. ${ }^{11}$ Note that the samples prepared at $1.2 \mathrm{M} \mathrm{KBr}$ with 0 and $0.1 \mathrm{M}$ added salt 

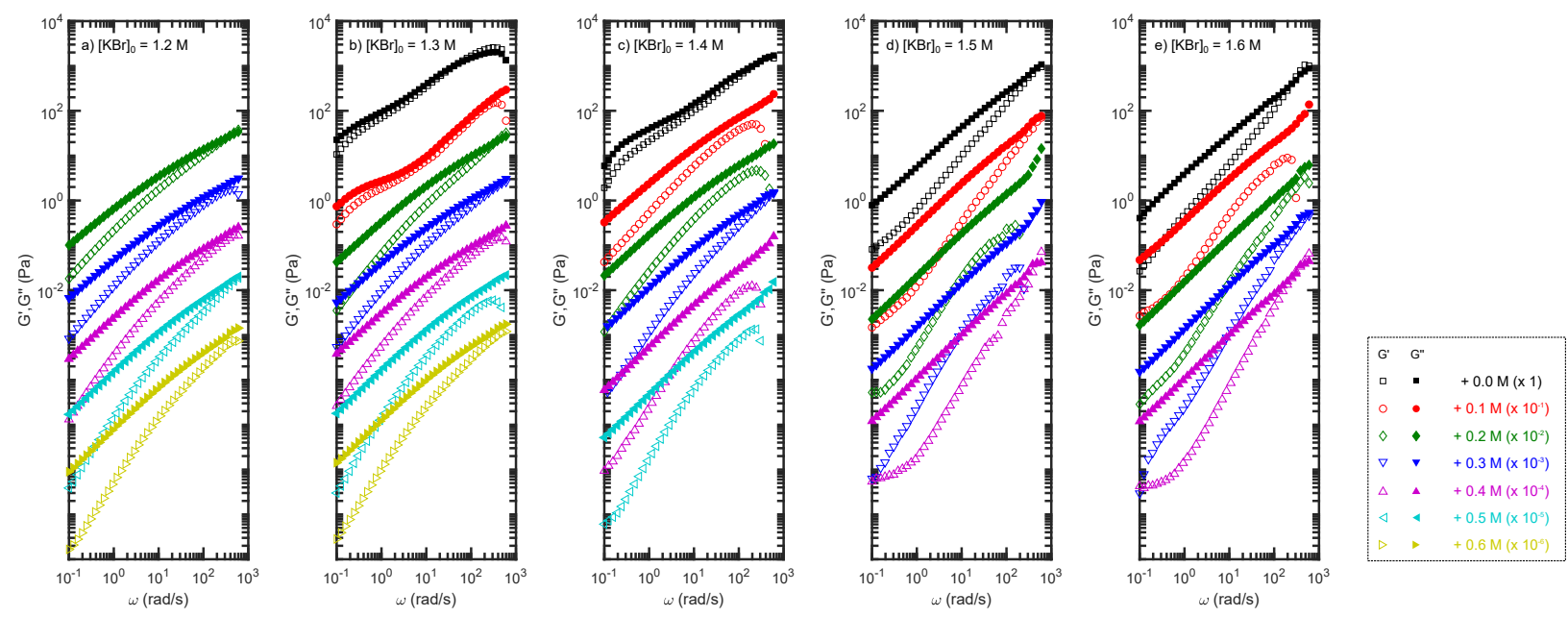

Figure 4. Frequency sweeps on samples prepared from coacervates with initial $\mathrm{KBr}$ concentrations of (a) $1.2 \mathrm{M}$, (b) $1.3 \mathrm{M}$, (c) $1.4 \mathrm{M}$, (d) $1.5 \mathrm{M}$, and (e) 1.6 M. In each plot, the as-prepared coacervate is shown in black, and samples with added salt are offset for visual clarity. Plots of the same data without this offset are included in the Supporting Information.

were omitted from the rheology experiments because they were too gel-like to obtain a satisfactory sample load on the rheometer with the limited sample volume available.

Upon addition of salt, the moduli of the samples decreased, and crossover points, when visible, shifted to slightly higher frequencies. The gel-like plateau observed in samples prepared at 1.3 and 1.4 $\mathrm{M}$ initial salt concentrations also disappeared with the addition of $0.2 \mathrm{M}$ or more $\mathrm{KBr}$, and all samples approached terminal flow in the limit of $0.5-0.6 \mathrm{M}$ added salt. We note that due to the low viscosities of the samples, some of the measurements did exhibit signatures of low torque errors (deviation of $G^{\prime}$ from $\omega^{2}$ scaling and significant noise in the amplitude sweeps at low frequency) and inertial limits (downturn of $G^{\prime}$ at high frequency), ${ }^{24,27}$ which limit the useful frequency range to approximately 2 decades in some samples; this limitation was unavoidable in the present experiments given the low sample volumes and available measurement geometries, but could be overcome in future experiments with altered sample preparation methods that allow large enough volumes of coacervates for use in sample geometries more appropriate for low-viscosity liquids.

The effect of added salt on relaxation times was quantified via time-salt superposition of the frequency sweep data. The samples prepared at 1.3 and $1.4 \mathrm{M} \mathrm{KBr}$ without added salt were omit- 


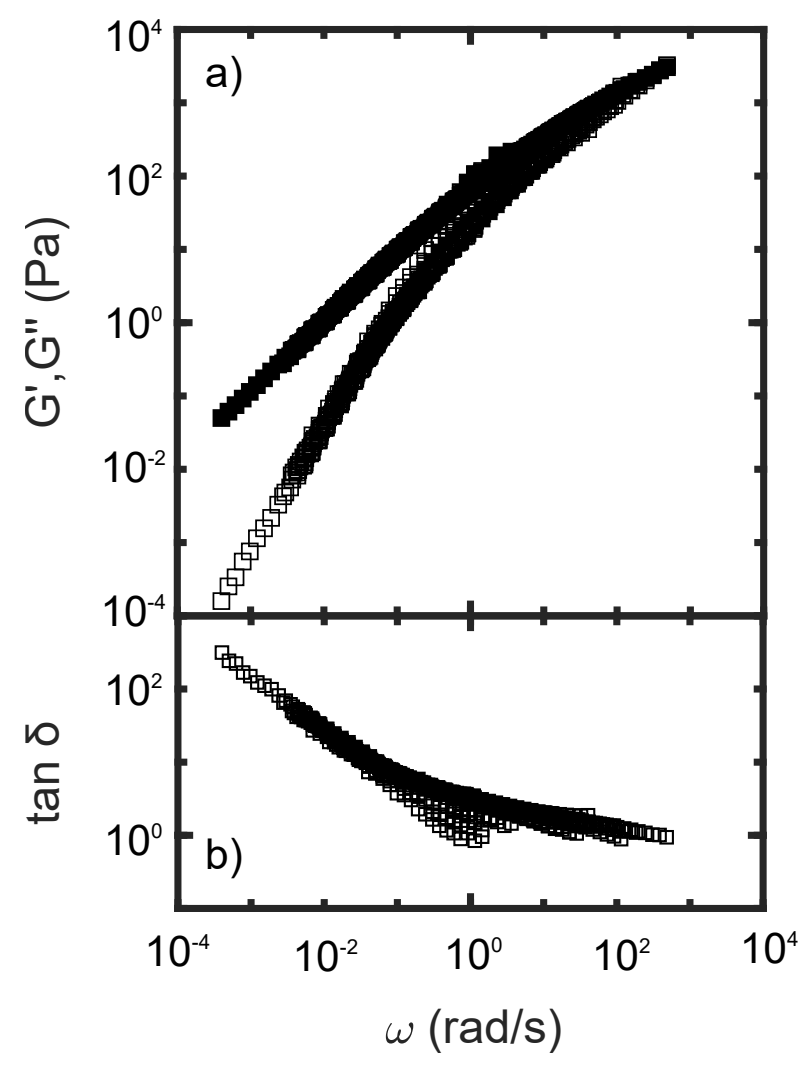

Figure 5. Superposed frequency sweeps on all samples included in the shift factor analysis. For convenience, the sample prepared at $1.2 \mathrm{M}$ salt with $0.2 \mathrm{M}$ added $\mathrm{KBr}$ was used as the reference trace for all samples, as it exhibited high quality data across the measured frequency range. The deviations from the master curve in $\tan \delta$ near $\omega=1 \mathrm{rad} / \mathrm{s}$ arise primarily from the samples prepared at an initial salt concentration of $1.6 \mathrm{M} \mathrm{KBr}$; individual plots separated by starting salt concentration illustrating this point are included in the Supporting Information.

ted from the superposition analysis, as was the sample prepared at $1.3 \mathrm{M}$ with $0.1 \mathrm{M}$ added $\mathrm{KBr}$, because the deviation from terminal flow in the gel-like plateau was too pronounced to allow for satisfactory superposition with the rest of the data. The remaining data were cropped to remove regions dominated by low-torque and inertial limits prior to superposition. The resulting superposed data are shown in Figure 5. As seen in this data, aside from slight deviations near the crossover point in samples prepared at an initial salt concentration of 1.6 M (see Supporting Information), satisfactory time-salt superposition was obtained across all samples.

The horizontal shift factors extracted from this analysis are plotted against both volume fraction of salt $\left(\phi_{\text {salt }}\right)$ and volume fraction of polymer $\left(\phi_{\text {pol }}\right)$ in Figure 6. In Figure 6(a), each data series 

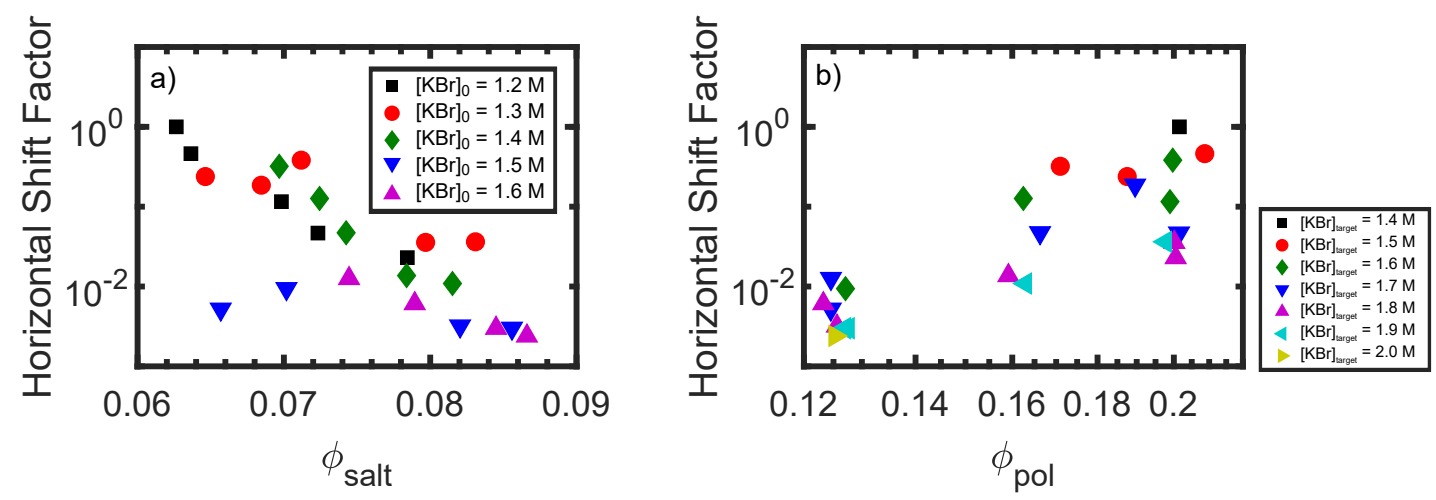

Figure 6. Horizontal shift factors obtained from superposition of all rheology data as a function of (a) volume fraction of salt $\left(\phi_{\text {salt }}\right)$ and (b) volume fraction of polymer $\left(\phi_{\text {pol }}\right)$. The sample prepared at $1.2 \mathrm{M}+0.2 \mathrm{M} \mathrm{KBr}$ was used as the reference trace, and has a horizontal shift factor of 1 .

represents samples prepared at the same initial salt concentration, and data within the series share a common value of $\phi_{p o l}$. In Figure 6(b), each data series represents samples with the same target salt concentration, and data within each series share, to a first approximation, a common value of $\phi_{\text {salt }}$. As seen in this figure, the shift factors, and thus relaxation times of the coacervates, decrease with increasing $\phi_{\text {salt }}$ and increase with increasing $\phi_{p o l}$. To quantify this dependence, shift factors with constant $\phi_{p o l}$ were fit to the functional form $a_{s} \sim e^{-B \phi_{\text {salt }}^{C} \text {, while shift factors for samples with }}$ the same target salt concentration were fit to the functional form $a_{s} \sim \phi_{\text {pol }}^{\beta}$. While the former fits did not yield a unique value for the exponent $C$ describing the salt-dependence of the activation energy for ion-pair rearrangement (see Supporting Information), likely due to the limited range of salt concentrations accessed in the present work, the latter fits generally indicated that the scaling exponent for the dependence of relaxation time on $\phi_{p o l}$ is between 3 and 6 , significantly higher than assumed in previous work. To verify that these large scaling exponents were not simply an effect of variation of $\phi_{\text {salt }}$ within each series of data targeting the same final salt concentration, the data were also grouped by the measured value of $\phi_{\text {salt }}$ obtained from TGA; repeating the analysis on these data gave similar scaling exponents, in the range of 3 to 6.5. $\phi_{\text {pol }}$ scaling exponents from both sets of fits are summarized in Table 1. 


\begin{tabular}{cccc}
\hline$[\mathrm{KBr}]_{\text {target }}(\mathrm{M})$ & $\beta$ & $\phi_{\text {salt }}$ & $\beta$ \\
\hline 1.5 & 1.9 & $0.070 \pm 0.0005$ & 6.5 \\
1.6 & 6.7 & $0.074 \pm 0.0005$ & 4.6 \\
1.7 & 5.2 & $0.079 \pm 0.0005$ & 3.2 \\
1.8 & 3.9 & & \\
1.9 & 5.6 & & \\
\hline
\end{tabular}

Table 1. Scaling exponents for fits to $a \sim \phi_{p o l}^{\beta}$ for samples prepared at different final salt concentrations

\section{Discussion}

The goals of this work were (1) to develop a method for preparing coacervate samples with varying salt concentrations without changing their polymer concentrations, and (2) to use this method to independently investigate how the relaxation times of coacervates depend on both polymer and salt. As shown in the Results, above, this effort was largely successful. The salt addition method indeed allowed samples to be prepared with varying salt concentrations at the same polymer concentration, with the polymer concentration of samples prepared from the same bulk coacervate typically varying by less than $10 \%$ while accessing salt concentrations up to $0.6 \mathrm{M}$ higher than the salt concentration of the initial coacervate. By adding salt directly to the coacervates after separating them from their supernatants, the composition of the samples was moved off the binodal and into the single-phase region of the phase diagram, which successfully decoupled the polymer concentration of the samples from the salt concentration at which they were prepared. Although samples prepared in this region of the phase diagram are most correctly described as single-phase solutions, we refer to them here as coacervates both for simplicity and to recognize the origins of the materials from which they were prepared.

While the primary goal of the TGA analysis was to assess the success of the salt-addition method in generating samples in which the salt and polymer concentrations are decoupled, as described above, two additional features of the composition data are worth noting. First, while the measured salt fractions of the samples prepared by salt addition were generally within $10 \%$ of that 
expected for salt solutions at the same molarity, the experimental numbers were systematically slightly lower than the theoretical estimates based on ideal mixing, as shown in Figure 2. This discrepancy likely results from non-idealities in the mixing of the samples, which has largely been omitted in phase diagrams generated by TGA to date ${ }^{21}$ however, our data indicate that the discrepancy is relatively small, and it is similarly ignored in the remainder of our analysis. Second, we also note that the tie lines connecting the as-prepared coacervates to their respective supernatants are generally relatively flat. Although a number of recent experimental and theoretical reports have suggested that the tie lines should slope downward, reflecting partitioning of the small salt ions into the supernatant phase due to excluded volume interactions, ${ }^{21}$ detailed analysis by Schlenoff et al. suggested that the tie line slope instead depends on the enthalpy of mixing. ${ }^{26}$ This work showed that mixing of PSS/PDADMAC is close to athermal in $\mathrm{KBr}$ solutions, which results in flat tie lines, ${ }^{26}$ as observed here.

The ability to prepare samples in which the salt and polymer concentrations are decoupled then allowed the contributions of these two factors to the relaxation times of the materials to be probed independently. First, the effect of changes in salt concentration at a constant polymer concentration was investigated by comparing the rheology of samples prepared by adding different amounts of salt to each initial bulk coacervate. Although the range of salt concentrations studied in this work was too narrow to uniquely extract the exponent describing the dependence of the activation energy for ion pair rearrangement on salt, the trends observed in this data provide strong evidence for the importance of salt-mediated interactions in complex coacervates. As seen in Figure 6(b), adding salt generally decreased the horizontal shift factor required for time-salt superposition, reflecting an increase in the relaxation rate. Interestingly, the magnitude of this change depended strongly on the salt concentration of the initial coacervate from which the samples were prepared. For the samples prepared by addition of salt to a coacervate originally prepared at $1.2 \mathrm{M} \mathrm{KBr}$, the shift factor gradually decreased by a factor of almost 100 over the $0.4 \mathrm{M}$ range of salt concentrations included in the analysis. For the samples prepared by addition of salt to coacervates with initial salt concentrations of 1.5 and 1.6 M KBr, however, the shift factors were far less sensitive to increases 
in salt concentration, changing by less than a factor of 10 over a similar range of salt concentrations. This observation suggests that the salt-dependent portion of the dynamics becomes saturated near 1.6 $\mathrm{M} \mathrm{KBr}$, which may reflect that this salt concentration is high enough to either fully screen ion-pairing interactions between chains, or shift the ion pairing equilibrium so strongly in favor of extrinsic charge compensation that further added salt does not have a significant impact on the number of inter-chain contacts. Interestingly, this salt concentration is also very close to the critical salt concentration for this polymer/salt system (approx. $1.8 \mathrm{M}$ ), ${ }^{3}$ suggesting that the saltmediated interactions that govern the relaxation timescales are also closely tied to those that drive coacervation.

The effect of changes in polymer concentration at a constant salt concentration was then investigated by comparing the relaxation times of samples with ether the same target salt concentration or the same volume fraction of salt as measured by TGA. When the horizontal shift factors were fit to the form $a_{s} \sim \phi_{p o l}^{\beta}$, the resulting scaling exponents were mostly in the range of 3-6, significantly higher than has been used in previous analyses of coacervate systems. ${ }^{11,14,17,18,21}$ Importantly, however, the appropriate scaling exponent depends strongly on the concentration regime in which the measurements are carried out. The sticky Rouse model predicts that when the volume fraction of polymer is higher than the overlap concentration of the strands between stickers, then in a good solvent the longest relaxation time should scale as $\phi^{0.15}$ if the chains are not entangled or $\phi^{1.44}$ if they are. When the volume fraction of polymer is lower than the overlap concentration of the strands between stickers, however, the dependence of the relaxation times on polymer fraction becomes significantly stronger, scaling as $\phi^{4.9}$ if the chains are not entangled, or as $\phi^{6.2}$ if they are. ${ }^{12,13}$ For the PSS/PDADMAC system at high salt concentrations, this low concentration limit may indeed be the relevant scaling regime. For a sample prepared at $1.6 \mathrm{M} \mathrm{KBr}$, for example, previous work has indicated that approximately one in ten charged monomers is engaged in intra-chain ion pairing interactions and functions as a "sticky" point. ${ }^{3}$ On average, there are thus approximately 10 repeat units between sticky points, which have an overlap concentration on the order of $60 \mathrm{wt} \%$ (see Supporting Information). The samples investigated in this work, however, typically 
have polymer concentrations on the order of 10-20 wt $\%$, placing them in the low concentration regime in which the scaling of relaxation time with polymer concentration is strong. Although this analysis breaks down at salt concentrations higher than $1.8 \mathrm{M}$ (where ion pairing analyses suggest that none of the charged sites continue to engage in intra-chain ion pairs and function as stickers ${ }^{3}$ ), and the variation in the experimental scaling exponents makes it difficult to perform a more quantitative comparison with this model, the strong polymer concentration dependence observed in this work is thus qualitatively consistent with the sticky Rouse model, and highlights the importance of choosing the correct concentration regime when analyzing coacervate relaxation rates.

The present salt-addition approach is thus a powerful way to decouple the otherwise correlated salt- and polymer-dependent relaxation behaviors of complex coacervates. Building on this work should offer exciting opportunities to investigate these properties over a wider range of compositions and polymer systems. Experiments on polymer systems such as poly(dimethylaminoethyl methacrylate) (PDMAEMA)/poly(acrylic acid) (PAA) that form liquid-like coacervates at lower salt concentrations may allow access to a wide enough range of salt concentrations to uniquely extract the salt-dependence of the activation energy for ion pair rearrangement. Preparation of samples by direct rehydration of dried coacervates to compositions targeting the single-phase region of the phase diagram may similarly allow access to a wider range of polymer and salt concentrations, and may also improve the accuracy with which target compositions can be prepared. Finally, extension to other polymer systems may allow investigation of how specific chemical and structural features of the polymers affect the salt-dependent dynamics of complex coacervates, independent of changes in polymer volume fraction. For example, investigation of salt-dependent dynamics in coacervates prepared from polymers with varying charge densities may provide insight into the role of cooperative interactions between neighboring charged sites on the polymer chains, while salt addition experiments using different salts added to the same starting coacervate may allow quantitative insight into ion-specific changes in ion pairing and relaxation dynamics in these systems. We expect that preparing and investigating samples above the binodal will provide fertile ground for further exploration and development of this versatile class of materials. 


\section{Conclusions}

We have shown that addition of salt to pre-formed polyelectrolyte complex coacervates allows the salt concentration of the coacervates to be changed without affecting the concentration of polymer. Using this approach, we investigated the salt- and polymer-dependent dynamics of coacervates of poly(styrene sulfonate) and poly(diallyldimethylammonium chloride). We found that the scaling of relaxation time with polymer fraction is significantly stronger, with scaling exponents between 3 and 6, than has been assumed to date, which may arise from the relatively low polymer concentrations in these samples relative to the concentrations necessary for overlap of the chains between stickers. This dependence on polymer volume fraction should be taken into account in physical analyses of the dynamics of these materials. More generally, we believe that salt addition experiments, and other experiments accessing compositions above the phase boundary, offer a route to new types of experiments on complex coacervates, and look forward to further work exploiting this technique to gain detailed physical understanding of these fascinating materials.

\section{Acknowledgments}

Acknowledgment is made to the Donors of the American Chemical Society Petroleum Research Fund for support of this research (Award No. 58034-DNI7). The authors also thank Yan Wang, Leanne Gilbertson, Anqi Wang, and Jung-Kun Lee for access to instrumentation used in this work.

\section{References}

[1] van der Gucht, J.; Spruijt, E.; Lemmers, M.; Stuart, M. A. C. Journal of Colloid and Interface Science 2011, 361, 407-422.

[2] Sing, C. E.; Perry, S. L. Soft Matter 2020, 16, 2885-2914.

[3] Wang, Q.; Schlenoff, J. B. Macromolecules 2014, 47, 3108-3116. 
[4] Liu, Y.; Winter, H. H.; Perry, S. L. Advances in Colloid and Interface Science 2016,

[5] Liu, Y.; Momani, B.; Winter, H. H.; Perry, S. L. Soft Matter 2017, 13, 7332-7340.

[6] Black, K. A.; Priftis, D.; Perry, S. L.; Yip, J.; Byun, W. Y.; Tirrell, M. ACS Macro Lett. 2014, 3, 1088-1091.

[7] Johnson, N. R.; Wang, Y. Expert Opin. Drug Deliv. 2014, 11, 1829-1832.

[8] Ahn, B. K.; Das, S.; Linstadt, R.; Kaufman, Y.; Martinez-Rodriguez, N. R.; Mirshafian, R.; Kesselman, E.; Talmon, Y.; Lipshutz, B. H.; Israelachvili, J. N.; Waite, J. H. Nat. Commun. 2015, 6 .

[9] Schaaf, P.; Schlenoff, J. B. Adv. Mater. 2015, 27, 2420-2432.

[10] Obermeyer, A. C.; Mills, C. E.; Dong, X.-H.; Flores, R. J.; Olsen, B. D. Soft Matter 2016, $12,3570-3581$.

[11] Spruijt, E.; Stuart, M. A. C.; van der Gucht, J. Macromolecules 2013, 46, 1633-1641.

[12] Rubinstein, M.; Semenov, A. N. Macromolecules 1998, 31, 1386-1397.

[13] Rubinstein, M.; Semenov, A. N. Macromolecules 2001, 34, 1058-1068.

[14] Yang, M.; Shi, J.; Schlenoff, J. B. Macromolecules 2019, 52, 1930-1941.

[15] Spruijt, E.; Sprakel, J.; Lemmers, M.; Stuart, M. A. C.; van der Gucht, J. Phys. Rev. Lett. 2010, 105.

[16] Sadman, K.; Wang, Q.; Chen, Y.; Keshavarz, B.; Jiang, Z.; Shull, K. R. Macromolecules 2017,

[17] Ali, S.; Prabhu, V. Gels 2018, 4, 11.

[18] Marciel, A. B.; Srivastava, S.; Tirrell, M. V. Soft Matter 2018, 14, 2454-2464. 
[19] Hamad, F. G.; Chen, Q.; Colby, R. H. Macromolecules 2018, 51, 5547-5555.

[20] Spruijt, E.; Westphal, A. H.; Borst, J. W.; Stuart, M. A. C.; van der Gucht, J. Macromolecules 2010, 43, 6476-6484.

[21] Li, L.; Srivastava, S.; Andreev, M.; Marciel, A. B.; de Pablo, J. J.; Tirrell, M. V. Macromolecules 2018, 51, 2988-2995.

[22] Andreev, M.; Prabhu, V. M.; Douglas, J. F.; Tirrell, M.; de Pablo, J. J. Macromolecules 2018, $51,6717-6723$.

[23] Huang, J.; Morin, F. J.; Laaser, J. E. Macromolecules 2019, 52, 4957-4967.

[24] Velankar, S. S.; Giles, D. Rheology Bulletin 2007, 76, 8-20.

[25] Fares, H. M.; Wang, Q.; Yang, M.; Schlenoff, J. B. Macromolecules 2018, 52, 610-619.

[26] Schlenoff, J. B.; Yang, M.; Digby, Z. A.; Wang, Q. Macromolecules 2019, 52, 9149-9159.

[27] Ewoldt, R. H.; Johnston, M. T.; Caretta, L. M. Complex Fluids in Biological Systems; Springer New York, 2014; pp 207-241. 